\title{
Variabilidade de Isolados de Microcyclus ulei no Sudeste da Bahia
}

\author{
Carlos R. R. Mattos ${ }^{1}$, Dominique Garcia ${ }^{2}$, Fabrice Pinard ${ }^{3} \&$ Vincent Le Guen ${ }^{3}$ \\ ${ }^{1}$ Plantações Michelin da Bahia, Cx. Postal 2, CEP 45435-000, Ituberá, BA, e-mail: carlos.rmattos@uol.com.br; ${ }^{2}$ Plantações E. \\ Michelin, Cx. Postal 80, CEP 78700-090, Rondonópolis, MT, e-mail: dominique.garcia@ cirad.fr; ${ }^{3}$ CIRAD-CP, Campus \\ Agronomique, BP/01, 97387 Kourou, Cedex, Guyane
}

(Aceito para publicação em 14/05/2003)

Autor para correspondência: Carlos R. R. Mattos

MATTOS, C.R.R., GARCIA, D., PINARD, F. \& LE GUEN, V. Variabilidade de isolados de Microcyclus ulei no Sudeste da Bahia. Fitopatologia Brasileira 28:502-507. 2003.

\section{RESUMO}

O fungo Microcyclus ulei, causador do mal-das-folhas da seringueira (Hevea brasiliensis), é o maior responsável pelo insucesso da heveicultura nas áreas tradicionais de cultivo no Brasil. Com o objetivo de conhecer melhor a diversidade desse patógeno foram estudados 50 isolados, obtidos nos anos 1997 e 1998, de uma área de 5.000 ha de seringueira da "Plantações Michelin da Bahia", no Sudeste da Bahia, através do tipo de reação de 12 clones de seringueira. As inoculações foram realizadas na face inferior dos folíolos jovens, repetidas três ou mais vezes em plantas diferentes, em câmara úmida com umidade superior a $95 \%$ e temperatura variando de 23 a $26^{\circ} \mathrm{C}$. Após 12 dias efetuaram-se as avaliações, utilizando-se uma escala de notas de 1 a 6 , que mede a virulência e a intensidade de esporulação. Foram identificados 36 padrões de virulência entre os 50 isolados testados, sendo que 21 apresentaram virulência a mais de nove clones e nenhum foi virulento a todos os 12 clones diferenciadores. A agressividade dos isolados, avaliada através da intensidade de esporulação, variou bastante com o clone. A exceção dos isolados FTP 11 e FTP 44, que de modo geral apresentaram uma agressividade fraca, um mesmo isolado apresentou-se altamente agressivo a um determinado clone e fracamente agressivo a outro. Alguns isolados, entretanto, mostraram uma alta agressividade aos clones de Hevea benthamiana, e outros aos clones de $H$. brasiliensis. Quatro isolados pareceram especializados sobre o clone FX 2784.

Palavras-chave adicionais: virulência, mal das folhas da seringueira.

\section{ABSTRACT}

Variability of Microcyclus ulei isolates from Southeast Bahia

South American Leaf Blight (SALB) of Hevea rubber (Hevea brasiliensis) tree, a disease caused by the fungus Microcyclus ulei, is the principal reason for the rather poor development of natural rubber production in Brazil. To obtain a more accurate knowledge of the diversity of this pathogen, 50 fungus isolates originated from the "Plantações Michelin da Bahia" were studied in 1997 and 1998, by observing the host's reaction type when inoculated on 12 different rubber clones. Inoculations were performed on the lower surface of young leaflets, with at least three replications in different plants and days, in an inoculation chamber with relative humidity superior to $95 \%$ and temperature between 23 and $26{ }^{\circ} \mathrm{C}$. Evaluation of symptoms was carried out 12 days after inoculation, using a 1 to 6 scale which measured virulence and sporulation intensity. Thirty-six perfils of virulence were identified among the 50 tested isolates, and 21 showed virulence on more than nine clones while none showed virulence on all 12 different clones. The aggressiveness of isolates, evaluated through the intensity of sporulation, varied significantly from one clone to another. Each isolate usually presented a high degree of aggressiveness to some clones and a low degree to to others, with the exception of isolates FTP 11 and FTP 44 which always displayed a low degree of aggressiveness. For example, some isolates showed higher aggressiveness to Hevea benthamiana clones, while others was more aggressive to H. brasiliensis clones. Four isolates seem to be specific to clone FX 2784.

\section{INTRODUÇÃO}

A produção de borracha natural no Brasil em 1996, segundo dados do IBAMA, apresentados por Hoelz (1999), foi de $53.438 \mathrm{t}$, ou seja, $43 \%$ da demanda nacional, num país que apresenta condições edafo-climáticas altamente favoráveis ao desenvolvimento da heveicultura e dispõe de milhões de hectares para plantio.

O insucesso da heveicultura (Hevea spp.) nas áreas tradicionais de cultivo no Brasil deve-se, principalmente, a ocorrência do mal das folhas da seringueira, causado pelo Microcyclus ulei, fungo originário da Amazônia, e que se encontra presente em todas as regiões heveícolas do país.
Essa doença, segundo Gasparotto et al (1984) e Silveira \& Furtado (1995), constituí-se em um dos principais fatores limitantes à expansão da heveicultura no Brasil, bem como em toda a América Latina (Holliday, 1970)

Embora o M. ulei tenha causado grandes prejuízos à heveicultura na Guiana (Bancroft, 1916) e no Suriname (Rogers \& Peterson, 1978) desde o início do século XX, somente a partir da década de 30, após a destruição de grande parte das plantações FORD no Brasil, iniciaram-se os trabalhos de pesquisa visando a seleção de clones resistentes ao $M$. ulei (Gonçalves, 1986).

O trabalho de seleção procedeu-se sem que houvesse um conhecimento maior das interações planta/patógeno, e 
dessa forma priorizou-se a resistência vertical, descobrindose mais tarde a ineficácia desse sistema devido ao aparecimento de raças fisiológicas, verificado inicialmente por Langford (1960) e confirmado posteriormente por Langdon (1965) e Miller (1966).

Embora em poucos trabalhos tenha havido preocupação com a avaliação da resistência horizontal em clones de seringueira [Hevea brasiliensis (Willd. Ex Adr. De Juss) Muell $\&$ Arj], sabe-se que a caracterização desse tipo de resistência é fundamental para um programa de seleção de clones (Peralta et al., 1990). Um avanço nesse sentido foi o estudo dos componentes da resistência horizontal da seringueira, desenvolvido por Junqueira et al. (1990) e Rivano (1997).

Outro fator importante a ser considerado num programa de melhoramento genético da seringueira para resistência ao M. ulei é o conhecimento da variabilidade genética desse patógeno (Junqueira, 1986). Nesse aspecto, alguns estudos já foram realizados (Miller, 1966; Brasil Sudhevea, 1975; Chee et al., 1986; Junqueira et al., 1986; Hashim \& Almeida, 1987; Furtado et al., 1995; Furtado, 1996; Rivano, 1997). Além destes, outros trabalhos deverão ser desenvolvidos para que se conheça melhor o comportamento desse fungo. Dessa forma, o presente trabalho teve como objetivo conhecer a variabilidade do M. ulei em uma plantação do Sudeste da Bahia.

\section{MATERIAL E MÉTODOS}

Foram estudados 50 isolados de M. ulei provenientes da "Plantações Michelin da Bahia", maior plantação de seringueira do Sudeste da Bahia, com área de 5.000 ha, através do tipo de reação em 12 clones de seringueira certificados através de eletroforese segundo Chevallier (1988). Esses clones foram escolhidos em função dos seus progenitores (Tabela 1), e com base nos trabalhos de Junqueira et al. (1986 e 1988).

Os isolados foram obtidos de 29 clones das espécies $H$. brasiliensis, $H$. benthamiana e $H$. pauciflora. As folhas infetadas foram coletadas em árvores do plantio industrial e nas coleções de clones, no período de 1992 a 1998.

As inoculações foram realizadas, com a ajuda de um atomizador (Paasche airbrusch set, modelo $\mathrm{H}$ ), na face inferior dos folíolos jovens e de coloração violeta, correspondentes ao estádio $\mathrm{B}_{2}$ descrito por Hallé \& Martin (1968), em câmara úmida com temperatura variando de 23 a $26^{\circ} \mathrm{C}$ e umidade superior a $95 \%$. Após as inoculações as plantas permaneceram 24 h no escuro, e a partir daí, até 12 dias, sob um regime de 12 $\mathrm{h}$ de escuro e $12 \mathrm{~h}$ de luz a 2000 lux.

Utilizou-se uma suspensão de $2 \times 10^{5}$ conídios $/ \mathrm{ml}$, acrescida de Tween 80 a $0,05 \%$, provenientes de lesões recém esporuladas, obtidas de clones sensíveis inoculados com isolados esporulados em meio de cultura segundo Mattos (1999).

As avaliações foram efetuadas em laboratório, 12 dias após as inoculações, em um estereomicroscópio, com um aumento de até 40 vezes, utilizando-se uma escala de 1 a 6 adaptada a partir da escala de Junqueira (1988), onde:

1 = Lesões necróticas sem esporos;

2 = Lesões não necróticas sem esporos;
3 = Esporulação muito fraca sobre a face inferior da lesão;

4 = Esporulação forte cobrindo parcialmente a face inferior da lesão;

$5=$ Esporulação muito forte cobrindo toda a face inferior da lesão;

6 = Esporulação muito forte cobrindo toda a face inferior da lesão e forte na face superior.

O trabalho foi realizado no período de janeiro a dezembro de 1997 e 1998, e as inoculações foram repetidas, pelo menos três vezes em plantas diferentes.

Para se ter uma imagem da diversidade da agressividade dos isolados foi realizada uma análise de correspondência (ACP), utilizando-se as notas de intensidade de esporulação dos clones testados como variáveis ativas através do software Winstat (CIRAD). $\tau$ representa a contribuição, em porcentagem, do eixo explicando o máximo da variação.

\section{RESULTADOS E DISCUSSÃO}

Dentre os 50 isolados testados, 36 diferiram na virulência a pelo menos um dos clones diferenciadores (Tabela 2). Os isolados mais virulentos esporularam em pelo menos dez dos 12 clones diferenciadores, enquanto os menos virulentos esporularam em apenas quatro desses clones. Os isolados FTP 2, 4, 6, 8, 13, 24, 29, PMB 5, 8, 15 e 16, foram os mais virulentos, infetando dez a 11 clones, seguidos dos isolados FTP 5, 17, 23, 45, 47, 50, 73, PMB 7, 9 e 12, infetando nove clones. Por outro lado, os isolados FTP 11, 14, 19, 20, 22 e 33, foram os menos virulentos, infetando quatro a seis clones.

Os isolados que apresentaram virulências semelhantes foram: FTP 2, FTP 4 e FTP 6; FTP 8, FTP 29, PMB 8 e PMB 15; FTP 17 e FTP 28; FTP 21 e PMB 4; FTP 22 e FTP 33; FTP 39, FTP 65, FTP 66 e PMB 10; FTP 55, PMB 2 e PMB 3; FTP 73 e PMB 9. Por outro lado, considerando a agressividade desses isolados, avaliada através da intensidade de esporulação (Tabela 3), todos diferiram entre si em pelo menos um dos clones testados.

É possível que as pequenas variações atribuídas à intensidade de esporulação tenham sido causadas por diferenças na idade dos folíolos, ou pela heterogeneidade genética do porta-enxerto, visto que foi considerada a maior nota das repetições em plantas diferentes. Apesar desta possível influência, pode-se observar através da análise de correspondência (Figura 1) uma organização das populações em função da agressividade dos isolados em 12 clones de seringueira das espécies $H$. brasiliensis, $H$. pauciflora e $H$. benthamiana. Os dois primeiros eixos contribuíram com 50,2\%, e o terceiro com $11,7 \%$ da variação geral. Os demais foram inferiores a $7 \%$. Dessa forma, somente os eixos 1 e 2 foram apresentados, sendo interpretados em função da posição dos clones diferenciadores (Figura 1-A). A Figura 1-B mostra a projeção das posições de cada isolado, sobre o plano constituído pelos eixos 1 e 2 .

A agressividade dos isolados se organizou segundo as espécies de Hevea (Figura 1-A), revelando quatro grupos distintos. O primeiro grupo reuniu os isolados especializados 
C.R.R. Mattos et al.

TABELA 1 - Clones de seringueira (Hevea brasiliensis) utilizados nos testes de patogenicidade de Microcyclus ulei

\begin{tabular}{|c|c|c|c|}
\hline Cloria & Proparitor & Fspócie de: Iteves & I ileratura \\
\hline $\mathrm{l} \times 2784$ & I: $4542 \times \wedge v 10=363$ & Hevera benthamiara $\mathrm{x}$ Je'vea brasilie flsis & \\
\hline $2 \times 3864$ & $1386 \times 1338$ & H. Irrasilientisis x $H$. brasilieflsis & 1 \\
\hline F'X 3899 & F $4542 \times$ Avlos 363 & 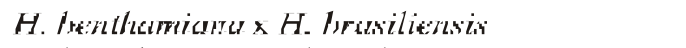 & $1: 2 ; 3$ \\
\hline $1 \times 985$ & I: $315 \times$ Aviths 183 & 11. Arusilientis x /I. brasiliefasis & $1 \div 2 ; 3 ; 4$ \\
\hline MIDI 180 & S lone prinuario & I1. Irasilientis & $1 \div 3$ \\
\hline$\left[{ }^{2} \wedge 3\right]$ & Slone primalio & H. paucijlora & $1 \div$ \\
\hline IA V 3087 & {$[\Gamma 4542 \times A v 108363] \times P B .86$} & [II, benihamiana x II. hrasiliensio $\mathrm{x}$ II brasil. & $1 \div 2$ \\
\hline IA V 717 & F $4542 \times$ PB 8,6 & I. benthamiona x II brasiliensis & $1 ; 2 ; 3 ; 4$ \\
\hline$\Gamma \times 2261$ & F $1619 \times$ Avrors 1883 & II, brasiliensis x II. brosiliensis & $1 ; 2 ; 3 \div 4$ \\
\hline$\Gamma \times 2804$ & F $4542 \times$ TJIR 1 & II, benlhamiana x II. brastiliensis & $1 ; 2 ; 3 \div 4$ \\
\hline$\Gamma \times 4098$ & PF $86 \times B 110$ & H, brosiliensis $\times$ II, brasiliensis & $1: 2 ; 3$ \\
\hline IA V 6158 & FX 43-655 x PB. 86 & {$[$ II, benlhomiana $\times$ II, brasiliensis] $\times$ II brasil. } & 1 \\
\hline
\end{tabular}

(1) Junqueira et al. (1986, 1988); (2) Rivano (1997); (3) Chee et al. (1986); (4) Hashim et al. (1987)

TABELA 2 - Reação de isolados de Microcyclus ulei em clones de seringueira (Hevea spp.)

\begin{tabular}{|c|c|c|c|c|c|c|c|c|c|c|c|c|c|}
\hline \multirow[b]{2}{*}{ Isulianlo } & \multicolumn{12}{|c|}{ Comportamento do elones } & \multirow[b]{2}{*}{ V III $=0$} \\
\hline & $\begin{array}{c}11 X \\
27 \times 4\end{array}$ & $\begin{array}{c}\text { IIX } \\
3 \times 64\end{array}$ & $\begin{array}{c}\text { I.X } \\
\mathbf{3 8 4 4}\end{array}$ & $\begin{array}{l}\operatorname{lix} \\
4 \pi 5\end{array}$ & $\begin{array}{c}\text { MIJP' } \\
\text { 180 }\end{array}$ & $\begin{array}{r}12 A \\
31\end{array}$ & $\begin{array}{l}\text { IAN } \\
\text { 3018K7 }\end{array}$ & $\begin{array}{l}\text { IAN } \\
717\end{array}$ & $\begin{array}{c}11 x \\
226,1\end{array}$ & $\begin{array}{c}1 . X \\
2 \times 144\end{array}$ & $\begin{array}{c}1 \times X \\
411198\end{array}$ & $\begin{array}{l}\text { IAN } \\
\text { h15K }\end{array}$ & \\
\hline FTP 14 & $\mathbf{R}$ & $\mathrm{s}$ & $\mathbf{R}$ & $\mathrm{R}$ & $\mathrm{s}$ & $\mathbf{R}$ & $\mathrm{s}$ & ? & $\mathrm{R}$ & $\mathrm{R}$ & $\mathrm{s}$ & $\mathrm{R}$ & 4 od 5 \\
\hline $\mathrm{F}=1 \mathrm{r} 22$ & R & 5 & R & $\mathrm{k}$ & 9 & $S$ & $\mathrm{k}$ & $\mathrm{s}$ & $\mathrm{I}$ & R & R & R & 1 \\
\hline FiT1 33 & R & s & R & $\mathrm{k}$ & $\mathrm{s}$ & $\mathrm{s}$ & $\mathrm{k}$ & $\mathrm{s}$ & $\mathrm{R}$ & R & R & $\mathrm{R}$ & 4 \\
\hline FTIR 11 & R & 5 & $s$ & $\mathrm{k}$ &. & R & $\mathrm{k}$ & $s$ & $\mathrm{~s}$ & $R$ & 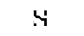 & $\mathrm{k}$ & $\delta$ \\
\hline $1+1 \% 19$ & R & 5 & R & $\$$ & $s$ & $s$ & 5 & R & $\mathrm{k}$ & R & $s$ & I & 6 \\
\hline $1.2 P 20$ & $R$ & 5 & $R$ & $s$ & $s$ & $s$ & 5 & $R$ & K & $R$ & $\mathrm{R}$ & 5 & 6 \\
\hline ISTP 7 & $\mathbf{R}$ & 5 & $s$ & $\$$ & $\mathrm{~s}$ & $\mathbf{R}$ & 5 & $\mathbf{R}$ & 5 & $\mathbf{R}$ & $\mathrm{s}$ & $\mathrm{R}$ & 7 \\
\hline FTP 18 & $\mathbf{R}$ & $\mathrm{s}$ & $\mathbf{R}$ & $\mathrm{R}$ & $\mathrm{s}$ & $?$ & 5 & $s$ & $\mathrm{~s}$ & $\mathrm{R}$ & $\mathrm{s}$ & 5 & 7 ous 8 \\
\hline $1: 11 \times 11$ & R & 5 & R & $\mathrm{k}$ & 9 & $S$ & 5 & R & R & $S$ & $S$ & $\varsigma$ & 7 \\
\hline FII 52 & R & s & R & 5 & $s$ & 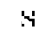 & $\mathrm{k}$ & $s$ & 5 & $s$ & R & $\mathrm{R}$ & $\%$ \\
\hline I'MIS I & R & 5 & R & $\$$ & 4 & R & 5 & $s$ & 5 & R & 4 & $\mathrm{R}$ & 7 \\
\hline PMIS 11 & R & 5 & $\mathrm{~s}$ & $\mathrm{k}$ & $s$ & R & 5 & $\mathrm{~s}$ & $\mathrm{k}$ & $\mathrm{s}$ & R & 5 & 7 \\
\hline PM⿻ 13 & $\mathbf{R}$ & 5 & $s$ & K & $S$ & R & $\mathrm{R}$ & $s$ & 5 & $R$ & $s$ & 5 & 7 \\
\hline FIF 3 & $\mathbf{R}$ & 5 & $s$ & $\mathbf{R}$ & $\mathrm{s}$ & $s$ & 5 & $s$ & 5 & $\mathrm{R}$ & $\mathrm{s}$ & $\mathrm{R}$ & 8 \\
\hline FTP 21 & $\mathbf{R}$ & $\mathrm{s}$ & $\mathbf{R}$ & 5 & $s$ & $s$ & $s$ & $s$ & $s$ & $\mathrm{R}$ & $\mathrm{s}$ & $\mathrm{R}$ & $\ddot{x}$ \\
\hline PMB 4 & $\mathrm{R}$ & $s$ & $\mathrm{R}$ & 5 & $s$ & $s$ & $s$ & $s$ & $\$$ & $\mathrm{R}$ & $s$ & $\mathrm{R}$ & 8 \\
\hline |:11P.39 & 3 & 5 & $s$ & $\mathrm{k}$ & 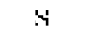 & R & 5 & R & 5 & $s$ & R & s & 8 \\
\hline FiTl &. & 5 & $\mathrm{~s}$ & $\mathrm{k}$ & .4 & R & 5 & R & $s$ & $s$ & R & S. & 8 \\
\hline F.T. 66 & $S$ & 5 & $s$ & $\mathrm{k}$ & $s$ & R & 5 & R & 5 & $s$ & R & 5 & 8 \\
\hline PMIS 10 & $s$ & 5 & $s$ & $1 F$ & $s$ & R & 5 & R & 5 & $s$ & R & 5 & 8 \\
\hline l'IP 55 & $\mathbf{R}$ & 5 & $\mathrm{~s}$ & $\mathrm{k}$ & $\mathrm{s}$ & $\mathbf{R}$ & 5 & $s$ & $\mathrm{R}$ & $\mathrm{s}$ & $\mathrm{s}$ & 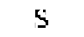 & 8 \\
\hline PMB 2 & $\mathbf{R}$ & $\varsigma$ & $s$ & $\mathrm{R}$ & $\mathrm{s}$ & $\mathbf{R}$ & 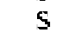 & $\mathrm{s}$ & $\mathrm{R}$ & $s$ & $\mathrm{~s}$ & $\varsigma$ & 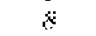 \\
\hline PMB 3 & $\mathrm{R}$ & $s$ & $s$ & $\mathrm{R}$ & $s$ & $\mathrm{R}$ & $s$ & $\$$ & $\mathrm{R}$ & $\mathrm{s}$ & $\mathrm{s}$ & $\mathrm{s}$ & is \\
\hline FTP 35 & $\mathrm{R}$ & 5 & $s$ & $\mathrm{~s}$ & $s$ & $\mathrm{~S}$ & $\mathrm{R}$ & $\mathrm{s}$ & $\mathrm{R}$ & $\mathrm{R}$ & $s$ & 5 & 8 \\
\hline$F I F .38$ & R & $s$ & R & 5 & $S$ & $S$ & $s$ & $s$ & R & $\mathrm{R}$ & $S$ & $s$ & $\dot{8}$ \\
\hline $1.10^{\circ} 58$ & R & 5 & $s$ & $\mathrm{k}$ & $s$ & $s$ & 5 & $\mathrm{~s}$ & $\mathrm{k}$ & R & $s$ & 5 & 8 \\
\hline PMע 6 & $R$ & 5 & $\mathbf{R}$ & $\mathbb{K}$ & $S$ & $S$ & 5 & $s$ & K & $s$ & $s$ & 5 & 8 \\
\hline$P M \amalg 14$ & $\mathbf{R}$ & 5 & $\mathbf{R}$ & $\mathrm{s}$ & $\mathrm{s}$ & $\mathrm{s}$ & 5 & $s$ & $\mathrm{R}$ & $s$ & $\mathrm{k}$ & 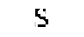 & $x$ \\
\hline FTP 5 & $\mathbf{R}$ & $s$ & $s$ & 5 & $s$ & $\mathrm{~s}$ & $s$ & $s$ & $s$ & $\mathrm{R}$ & $\mathrm{s}$ & $\mathrm{R}$ & 9 \\
\hline FTP 17 & $\mathrm{R}$ & $s$ & $\mathrm{~s}$ & 5 & $s$ & $\mathrm{R}$ & $s$ & $s$ & $s$ & $\mathrm{~s}$ & $\mathrm{R}$ & $s$ & 9 \\
\hline FTP 23 & $\mathrm{R}$ & 5 & $\mathrm{R}$ & $\mathrm{R}$ & $\mathrm{s}$ & $\mathrm{S}$ & $s$ & $S$ & 5 & $S$ & $s$ & $\mathrm{~s}$ & 9 \\
\hline $\mathrm{FIO} 12$ & R & $s$ & $s$ & 5 & $\$$ & R & $s$ & $s$ & R & $\mathrm{s}$ & $\$$ & $\mathrm{~s}$ & ب̣! \\
\hline $1: 11 \%$ & R & 5 & R & 5 & $\mathrm{~s}$ & $\mathrm{~s}$ & 5 & $s$ & 5 & R & 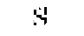 & 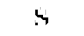 & 9 \\
\hline FTP 50 & R & 5 & $s$ & $\xi$ & $\mathrm{s}$ & $s$ & $s$ & $s$ & IR & $R^{2}$ & $\mathrm{~s}$ & 5 & 9 \\
\hline PMШ 7 & $\mathbf{R}$ & 5 & $\mathbf{R}$ & 3 & $\mathrm{~s}$ & $\mathrm{~s}$ & $\$$ & $\mathrm{~s}$ & $s$ & $\mathrm{~s}$ & $\mathrm{~s}$ & $\mathrm{R}$ & 9 \\
\hline PMB 12 & $\mathbf{R}$ & $\mathrm{s}$ & $\mathrm{s}$ & $\mathrm{R}$ & $\mathrm{s}$ & $\mathrm{s}$ & 5 & $s$ & $\mathrm{~s}$ & $s$ & $\mathrm{R}$ & 5 & 9 \\
\hline FTP 73 & $\mathrm{R}$ & $s$ & $\mathrm{~s}$ & $\mathrm{R}$ & $\mathrm{s}$ & $\mathrm{R}$ & $s$ & $s$ & $s$ & $\mathrm{~s}$ & $\mathrm{~s}$ & $\$$ & 9 \\
\hline PMA 9 & $\mathrm{R}$ & 5 & $S$ & $\mathrm{~K}$ & $S$ & $\mathrm{R}$ & $s$ & $S$ & $s$ & $\mathrm{~S}$ & $s$ & 5 & 9 \\
\hline $\mathrm{FII} 1 \%$ & R & 5 & $s$ & 5 & 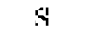 & $\mathrm{R}$ & 5 & $s$ & 5 & $s$ & R & 5 & 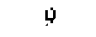 \\
\hline $1: 11^{+} 25$ & R & s & $s$ & 5 & 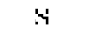 & R & 5 & $s$ & $?$ & $s$ & R & s & 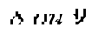 \\
\hline $1: 11^{1} 2$ & R & 5 & $s$ & 5 & .4 & .9 & 5 & $\mathrm{~s}$ & 5 & R & .4 & 5 & lo \\
\hline IIT 4 & $\mathbf{R}$ & $\$$ & $s$ & $\mathrm{~s}$ & $\mathrm{~s}$ & $\mathrm{~s}$ & 5 & $s$ & $s$ & $\mathbf{R}$ & $\mathrm{s}$ & 5 & 10 \\
\hline FTP 6 & $\mathbf{R}$ & $\mathrm{s}$ & $s$ & 5 & $s$ & $\mathrm{~s}$ & $\mathrm{~s}$ & $s$ & $\mathrm{~s}$ & $\mathrm{R}$ & $\mathrm{s}$ & $\mathrm{s}$ & 10 \\
\hline FTP 8 & $\mathrm{R}$ & 5 & $\mathrm{R}$ & 5 & 5 & $s$ & 5 & $s$ & 5 & $\mathrm{~s}$ & $s$ & 5 & 10 \\
\hline FTP 29 & $\mathrm{R}$ & $\varsigma$ & $\mathrm{R}$ & $\mathrm{s}$ & $\mathrm{s}$ & $\mathrm{S}$ & $\xi$ & $\mathrm{S}$ & 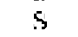 & $\mathrm{s}$ & $\mathrm{s}$ & 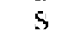 & 10 \\
\hline P.MIS \& & R & $\$$ & R & 5 & $\$$ & $\$$ & $\$$ & $\$$ & $\$$ & $\$$ & $\$$ & $\$$ & 10 \\
\hline PMIS 1S & R & 5 & R & 5 & $\mathrm{~S}$ & $\mathrm{~s}$ & 5 & $\mathrm{~s}$ & 5 & $\mathrm{~s}$ & 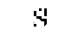 & ל & 10 \\
\hline IPMI5 5 & R & 5 & $s$ & $\mathrm{k}$ & $\stackrel{4}{4}$ & 4 & 5 & $\stackrel{9}{9}$ & 5 & $s$ & 4 & 5 & (i) \\
\hline $1:\left.T\right|^{2}>4$ & R & 5 & $s$ & $s$ & 9 & R & 5 & $S$ & 5 & $\$$ & .9 & 5 & 16 \\
\hline PMB 16 & $\mathbf{R}$ & $\mathrm{s}$ & $s$ & 5 & $\mathrm{~s}$ & $\mathrm{~s}$ & $s$ & $s$ & $\mathrm{R}$ & $s$ & $\mathrm{~s}$ & $s$ & 10 \\
\hline FTP 13 & $\mathrm{R}$ & $s$ & $\mathrm{~s}$ & 5 & $s$ & $\mathrm{~s}$ & $s$ & $s$ & $s$ & $s$ & $s$ & $\$$ & 11 \\
\hline
\end{tabular}

*S = Suscetível (com esporulação); $\mathrm{R}$ = Resistente (sem esporulação); ? Não realizado;

$* * \mathrm{VIR}=\mathrm{N}^{\circ}$ de virulências 
TABELA 3 - Notas de intensidade de esporulação de isolados de Microcyclus ulei em clones de seringueira (Hevea spp.)

\begin{tabular}{|c|c|c|c|c|c|c|c|c|c|c|c|c|}
\hline \multirow{2}{*}{ Isolado } & \multicolumn{12}{|c|}{ Comportamento diss elones } \\
\hline & FX 27\&4 & FX 3\&rit & FX 389y & FX 985 & MDF $1 \$ 0$ & PA 31 & IAN 3087 & ISN 717 & FX 2261 & FX 2804 & FX 4098 & IAN 6158 \\
\hline FiTP & 2 & 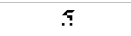 & 3 & $s$ & 5 & 3 & 4 & 3 & 3 & 1 & 4 & 3 \\
\hline lip 3 & 1 & 6 & 3 & 1 & 4 & 4 & 3 & 3 & 3 & 1 & 4 & 1 \\
\hline FTP 4 & 2 & 6 & 3 & 5 & 5 & 3 & 4 & 3 & 5 & 1 & 4 & 3 \\
\hline FTP 5 & 1 & 6 & 3 & 3 & 4 & 4 & 3 & 3 & 3 & 1 & 1 & 1 \\
\hline Fip & 1 & 6 & $\xi$ & 4 & 4 & 4 & 5 & $<$ & 3 & 1 & 4 & 3 \\
\hline IPTP 7 & 1 & 4 & $<$ & 4 & 5 & 1 & 4 & $:$ & 4 & 1 & 5 & 1 \\
\hline FTP \& & 2 & 5 & 1 & 3 & 4 & 3 & 4 & 3 & 4 & 3 & 3 & 3 \\
\hline 11 & 1 & 1 & 3 & 1 & 4 & 1 & 1 & 3 & 3 & 1 & 1 & 1 \\
\hline 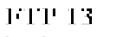 & 2 & 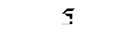 & $<$ & 3 & 4 & 3 & 6 & 5 & 4 & 5 & 3 & 6 \\
\hline FIP 14 & 1 & 6 & 1 & 1 & 4 & 1 & 4 & ?? & 1 & 1 & 5 & 1 \\
\hline FTP 17 & 2 & 4 & 5 & 5 & 4 & 1 & 5 & $<$ & 3 & 4 & 2 & 4 \\
\hline FTT' 18 & 1 & 6 & 1 & 1 & 4 & $?$ & 1 & 1 & 4 & 1 & 6 & 1 \\
\hline FITI 14 & 1 & 6 & 1 & 6 & 5 & 4 & 6 & : & 1 & 1 & 4 & 1 \\
\hline FTP 20 & 2 & 6 & 1 & 3 & 5 & 4 & 5 & 2 & 2 & 2 & 2 & 3 \\
\hline FTT' 21 & 1 & 6 & 1 & 1 & 4 & 4 & 6 & 1 & 4 & 1 & 1 & 2 \\
\hline 329 & 2 & 4 & 1 & 1 & 5 & 3 & 4 & 3 & 3 & 3 & 3 & 3 \\
\hline כרבין & 1 & 5 & 1 & 1 & 4 & 3 & 1 & 3 & 1 & 1 & 1 & 1 \\
\hline FTP 24 & 2 & 5 & 5 & 4 & 5 & 2 & 5 & $<$ & 4 & 3 & 4 & 6 \\
\hline FTP 28 & 1 & 6 & 6 & 6 & 4 & 1 & 6 & 6 & $?$ & 3 & 1 & 5 \\
\hline FII 20 & 1 & 6 & 1 & 1 & 1 & 1 & 1 & , & 1 & 3 & 6 & 1 \\
\hline 1913 & 1 & 6 & 1 & 1 & 4 & 3 & 1 & 3 & 1 & 1 & 1 & 1 \\
\hline FTP 35 & 1 & 4 & $\angle$ & 6 & 4 & 4 & 1 & 3 & 1 & 1 & 3 & 6 \\
\hline FII 38 & 1 & 6 & 1 & 1 & 4 & 3 & 1 & 3 & 1 & 1 & 3 & 3 \\
\hline צ'ו & 6 & 4 & $<$ & 1 & 4 & 1 & 3 & : & 4 & 3 & 1 & 4 \\
\hline IF'P 44 & 2 & 4 & 2 & 1 & 4 & 3 & 4 & $:$ & 2 & 3 & 4 & 3 \\
\hline FTJ 45 & 2 & 5 & 3 & 4 & 4 & 2 & 5 & 3 & 2 & 3 & 4 & 3 \\
\hline FII & 1 & 6 & 1 & 6 & 4 & 1 & 6 & 1 & 6 & 1 & 1 & 1 \\
\hline 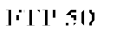 & 1 & 6 & ; & 6 & 4 & 4 & 4 & ; & 1 & 1 & 4 & 3 \\
\hline FIP 52 & 1 & 6 & 1 & 4 & 4 & 4 & 1 & 3 & 3 & 3 & 1 & 1 \\
\hline FTT 55 & 1 & 1 & ii & 1 & 4 & 1 & 6 & 1 & 1 & 6 & 1 & 5 \\
\hline 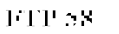 & 1 & 6 & 3 & 1 & 4 & 4 & 3 & 3 & 1 & 1 & 6 & 3 \\
\hline FiTP & 6 & 4 & $<$ & 1 & 4 & 1 & 4 & : & 4 & 3 & 1 & 4 \\
\hline FTP 66 & 6 & 4 & $<$ & 1 & 4 & 1 & 4 & : & 4 & 3 & 1 & 4 \\
\hline FTP 73 & 1 & 5 & 1 & 1 & 4 & 1 & 5 & 1 & 6 & 4 & 1 & 3 \\
\hline PMIt 1 & 1 & 1 & 1 & 6 & 4 & 1 & 3 & 3 & 1 & 1 & 3 & 1 \\
\hline lPMIl $)$ & 1 & 4 & 5 & 1 & 4 & 1 & 6 & 5 & 1 & 6 & 4 & 5 \\
\hline PMH 3 & 1 & 3 & 5 & 1 & 4 & 1 & 6 & 5 & 1 & 5 & 4 & 5 \\
\hline P:MIt 1 & 1 & 6 & 1 & 3 & 4 & 1 & 3 & 3 & 1 & 1 & 1 & 1 \\
\hline I"MIl ૬ & 2 & 6 & ; & I & 5 & 4 & 3 & $<$ & 3 & 4 & 4 & 4 \\
\hline PML 6 & 1 & 5 & 1 & 1 & 4 & 4 & 5 & $<$ & 1 & 3 & 5 & 3 \\
\hline PME 7 & 1 & 5 & 1 & 5 & 4 & 3 & 4 & 3 & 4 & 4 & 4 & 1 \\
\hline TMF \& & 1 & 6 & 1 & 1 & 1 & 1 & 1 & i & 1 & 3 & 3 & 3 \\
\hline IיMII & 2 & 6 & $<$ & 1 & 5 & 1 & 3 & ; & 6 & 3 & 4 & 3 \\
\hline PMI 10 & 6 & 4 & $<$ & 1 & 4 & 1 & 3 & : & 4 & 3 & 1 & 3 \\
\hline PMB 11 & 2 & 4 & G & 1 & 4 & 1 & 6 & 0 & 1 & i & 1 & 6 \\
\hline TMB 12 & 2 & 6 & 3 & 1 & 5 & 3 & 3 & i & 5 & 3 & 2 & 3 \\
\hline PMII 13 & 2 & 4 & $\ddot{3}$ & 1 & 4 & 7 & 7 & $<$ & 3 & 7 & 3 & 6 \\
\hline PMB 14 & 2 & 6 & ] & 5 & 4 & 3 & 5 & $<$ & 2 & 3 & 2 & 3 \\
\hline TMB 15 & 2 & 6 & 1 & 3 & 5 & 1 & 3 & 3 & 4 & 3 & 6 & 3 \\
\hline P.MH 16 & 2 & $\therefore$ & h & 1 & $s$ & 1 & 6 & 5 & 2 & $s$ & 1 & 6 \\
\hline
\end{tabular}

* Notas: 1 e 2: lesões sem esporos; 3 e 4: lesões com esporulação fraca; 5 e 6: lesões com esporulação forte e muito forte, respectivamente. ? Não realizado.

no clone FX 2784: PMB 10, FTP 39, FTP 66, FTP 65 (Figura 1B). A esporulação desses isolados no FX 2784 ocorreu no nível máximo da escala de intensidade de esporulação, ou seja, muito forte sobre as duas faces do folíolo. Por outro lado, nos demais clones, inclusive nos clones orientais que são altamente suscetíveis ao M. ulei, a esporulação foi muito fraca. Tal fato sugere que esses isolados, talvez, pertençam a uma raça nova altamente especializada. O segundo grupo se caracterizou por uma alta agressividade aos clones de $H$. benthamiana: PMB 11, FTP 55, PMB 3, PMB 2, PMB 16 (Figura 1-B). O terceiro grupo, representado pela maioria dos isolados, foi mais agressivo aos clones de $H$. brasiliensis, e o quarto grupo, que aparece no centro da Figura 1-B, apresentou uma agressividade elevada tanto aos clones de $H$. brasiliensis quanto aos clones de H. benthamiana. Demonstrou-se, assim, uma especificidade para hospedeiros mais sob o aspecto quantitativo do que qualitativo como foi observado por Junqueira et al. (1986).

Essa grande variabilidade dos isolados de $M$. ulei em uma plantação de seringueira (Hevea spp) no Sudeste da Bahia confirma os resultados obtidos por Junqueira et al. (1986) e Rivano (1997), e pode estar associada à reprodução sexuada. Segundo Camargo (1995), os fungos que se reproduzem sexuadamente estão entre os que apresentam maior variabilidade fitopatogênica.

Chee et al. (1986) identificaram as raças 1, 2, 4, 5, 6, 7, 8, e 9 de M. ulei no Estado da Bahia, acrescentando, portanto, cinco raças as quatro identificadas por Miller (1966). As raças 2, 4, 5, e 6 também foram identificadas por Hashim \& Almeida 


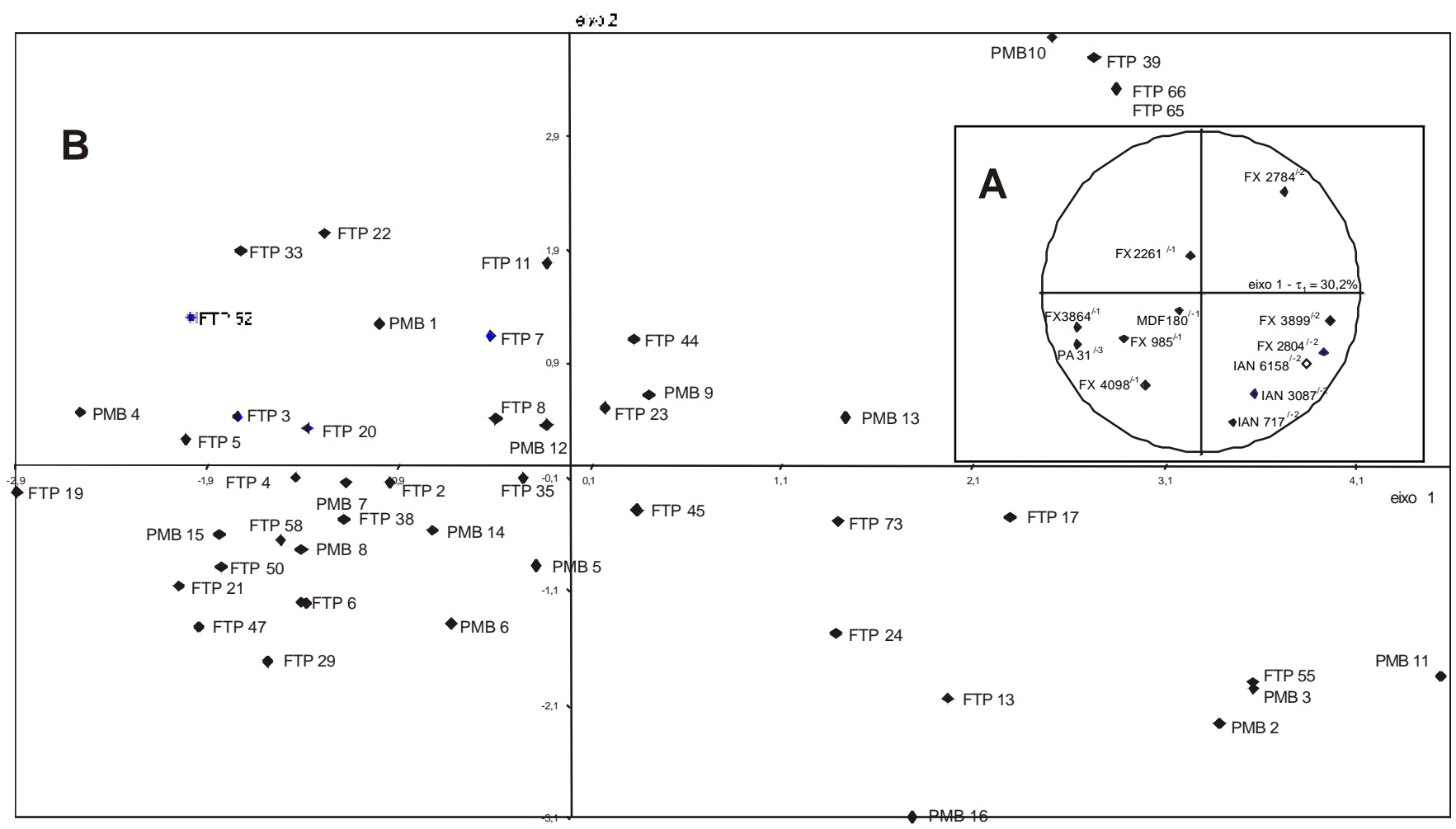

/-1 Hevea brasiliensis; /-2 Hevea benthamiana; /-3 Hevea pauciflora

FIG. 1 - Análise de correspondência (ACP) realizada a partir dos dados da Tabela 2. A/ Representação das variáveis ativas "notas de intensidade de esporulação sobre os clones diferenciadores”. B/ Distribuição dos isolados segundo a análise de correspondência (ACP).

(1987), estudando 68 isolados de M. ulei. Apenas os isolados FTP 7, PMB 11, FTP 20 e FTP 19, se enquadram nas raças 5, 2, 7, e 9, respectivamente, considerando o tipo de reação de apenas seis clones (IAN 717, FX 985, FX 2261, FX 2804, FX3899 e FX 4098), comuns aos dois trabalhos (Tabela 2). Por outro lado, se forem considerados os resultados obtidos por Junqueira $e t a l$. (1986), com 16 isolados de $M$. ulei de várias regiões do país, apenas os isolados EIR e BEN poderiam ser comparados a dois dos 36 isolados fisiologicamente diferentes obtidos no presente trabalho, ou seja, ao PMB 1 e ao FTP 47, respectivamente.

Na Guiana Francesa, Rivano (1997), trabalhando com 16 isolados de $M$. ulei, provenientes de uma área experimental no interior da floresta, detectou a presença de 11 raças diferentes. Com relação ao presente trabalho, as únicas semelhanças observadas foram em relação aos isolados $\mathrm{G} 2, \mathrm{G}$ 5 e G 35 com os isolados FTP 19, FTP 20, e FTP 14, respectivamente.

Embora os trabalhos desenvolvidos por Junqueira et al. (1986) e Rivano (1997) tenham demonstrado uma grande variabilidade do $M$. ulei, o presente trabalho avançou nesse aspecto, e se destaca por ter utilizado um grande número de isolados obtidos de uma única plantação de seringueira.

A metodologia utilizada no presente trabalho foi semelhante a dos demais autores citados, à exceção de Chee et al. (1986) e Hashim \& Almeida (1987) que trabalharam com discos de folhas. Porém, o número de clones diferenciadores comuns a todos os trabalhos foi bastante pequeno e, possivelmente, insuficiente para diferenciar aqueles isolados que apresentaram comportamento semelhante.

Dessa forma, acredita-se que os isolados estudados pelos diferentes autores se constituam em raças diferentes de M. ulei, e que, pelo menos, 36 dos 50 isolados estudados no presente trabalho possam tratar-se de novas raças do patógeno. A grande diversidade do poder patogênico desse parasita no Sudeste da Bahia mostra a sua elevada capacidade de adaptação e a dificuldade para se obter, no futuro, clones com resistência durável.

\section{REFERÊNCIAS BIBLIOGRÁFICAS}

BANCROFT, C.K. Report on the South American leaf disease of the rubber tree. Journal of Board Agriculture 10:13-33. 1916.

BRASIL SUDHEVEA. Plano Nacional da Borracha. Pesquisas Fitopatológicas. Pesquisas e experimentação realizadas com a seringueira. Rio de Janeiro, (Anexo II). 1975. pp.36-107.

CAMARGO, L.E.A. Genética da Interação Hospedeiro-Patógeno. In: Bergamin Filho, A., Kimati, H. \& Amorim, L. (Eds.) Manual de Fitopatologia, v. 1: Princípios e Conceitos. São Paulo, Agronômica Ceres, 1995. pp.454-469.

CHEE, K.H., KAI-MING, Z. \& DARMONO, T.W. Occurrence of eight races of Microcyclus ulei on Hevea rubber in Bahia, Brasil. Transactions of the British Mycological Society 87:15-21. 1986.

CHEVALLIER, M.H. Genetic variability of Hevea brasiliensis 
germplasm using isozyme markers. Journal of Natural Rubber Research 3:42-53. 1988.

FURTADO, E.L., MENTEN, J.O.M., SILVEIRA, A.P. \& JUNQUEIRA, N.T.V. Nova raça de M. ulei (P. Henn.) V. Arx. na região do Vale do Ribeira-SP. Fitopatologia Brasileira 20:344. 1995. (Resumo)

FURTADO, E.L. Comportamento de cultivares de seringueira (Hevea spp.) frente ao mal das folhas na região do vale do Ribeira, SP. (Tese de Doutorado). Piracicaba. ESALQ. 1996.

GASPAROTTO, L., TRINDADE, D.R. \& SILVA, H.M. Doenças da seringueira. Manaus, EMBRAPA/CNPSD, Circular Técnica, 4. 1984.

GONÇALVES, P.S. Melhoramento genético da seringueira (Hevea spp) Anais, Simpósio Sobre a Cultura da Seringueira no Estado de São Paulo. Piracicaba, 1986. pp.95-123.

HALLÉ, F. \& MARTIN, R. Étude de la croissance rythmique chez l'hévéa (Hevea brasiliensis Müll. Arg., Euphorbiacées, Crotonoïdées). Adansonia 8:475-503. 1968.

HASHIM, I. \& ALMEIDA, L.C.C. de. Identification of races and in vitro sporulation of M. ulei. Journal of Natural Rubber Research 2:111-117. 1987.

HOELZ, J.J. Subsídios sobre o histórico e expansão da produção e consumo da borracha natural. II Curso sobre a cultura da seringueira, UNESP, Botucatu, 1999. pp.1-45.

HOLLIDAY, P. South American leaf blight (M. ulei) of Hevea brasiliensis. Commonwealth Mycological Institute. Phytopathology Papers 12:1-31. 1970.

JUNQUEIRA, N.T.V., CHAVES, G.M., ZAMBOLIM, L., GASPAROTTO, L. \& ALFENAS, A.C. Variabilidade fisiológica de M. ulei. Fitopatologia Brasileira 11: 823-833. 1986.

JUNQUEIRA, N.T.V., CHAVES, G.M., ZAMBOLIM, L.,
ALFENAS, A.C. \& GASPAROTTO, L. Reação de clones de seringueira a vários isolados de M. ulei. Pesquisa Agropecuária Brasileira 23:877-893. 1988.

JUNQUEIRA, N.T.V., LIEBEREI, R., KALIL FILHO, A.N. \& LIMA M.I.P.M. Components of parcial resistance in Hevea clones to rubber tree leaf blight, caused by M. ulei. Fitopatologia Brasileira 15:211214. 1990.

LANGDON, K. R. Relative resistance or susceptibility of several clones of Hevea brasiliensis and Hevea brasiliensis x Hevea benthamiana to two races of Dothidella ulei. Plant Disease Reporter 49: 12-14. 1965.

LANGFORD, M.H. A new strain of leaf blight on rubber trees in Costa Rica. Washington, A. I. D., 1960.

MATTOS, C.R.R. Meios de cultura com água de coco verde para esporulação de Microcyclusulei. Fitopatologia Brasileira 24:470. 1999. (Resumo)

MILLER, J.W. Differencial clones of Hevea for identifying races of Dothidella ulei. Plant Disease Reporter. 50:187-190. 1966.

PERALTA, A.M., FURTADO. E.L., AMORIM, L., MENTEN, J.O.M. \& BERGAMIN FILHO, A. Melhoramento genético da seringueira para a resistência ao mal das folhas (Microcyclus ulei)Revisão. Summa Phytopathologica 16:214-224. 1990.

RIVANO, F. La maladie sud-américaine des feuilles de l'hévéa. I. Variabilité du pouvoir pathogène de Microcyclus ulei. Plantations, Recherche, Développement 4:104-110. 1997.

ROGERS, T.H. \& PETERSON, A.L. Control of South American leaf blight on a plantation scale in Brazil. Proceedings of the Internatioanal Rubber Conference. Kuala Lumpur, 1978. pp.266-277.

SILVEIRA, A.P. \& FURTADO, E.L. Doenças da Seringueira no Estado de São Paulo. São Paulo: Instituto Biológico, Boletim Técnico n.7. 1995. 